\title{
New Neural Network Corresponding to the Evolution Process of the Brain
}

\author{
Seisuke Yanagawa \\ OptID, Machida, Tokyo, Japan \\ Email address: \\ s_yanagawa@jcom.home.ne.jp
}

\section{To cite this article:}

Seisuke Yanagawa. New Neural Network Corresponding to the Evolution Process of the Brain. American Journal of Neural Networks and Applications. Vol. 7, No. 1, 2021, pp. 1-6. doi: 10.11648/j.ajnna.20210701.11

Received: January 21, 2021; Accepted: January 28, 2021; Published: February 9, 2021

\begin{abstract}
In this paper, the logic is developed assuming that all parts of the brain are composed of a combination of modules that basically have the same structure. The fundamental function is the feeding behavior searching for food while avoiding the dangers. This is most necessary function of animals in the early stages of evolution and the basis of time series data processing. The module is presented by a neural network with learning capabilities based on Hebb's law and is called the basic unit. The basic units are placed on layers and the information between the layers is bidirectional. This new neural network is an extension of the traditional neural network that evolved from pattern recognition. The biggest feature is that in the process of processing time series data, the activated part in the neural network changes according to the context structure of the data. Predicts events from the context of learned behavior and selects best way. It is important to incorporate higher levels of intelligence such as learning, imitation functions furthermore long-term memory and object symbolization. A new neural network that deals the "descriptive world" that expresses past and future events to the neural network that deals the "real world" related to the familiar events is added. The scheme of neural network's function is shown using concept of category theory
\end{abstract}

Keywords: Time-Series Data, Acceptance and Generation, Learning Context of Data, Prediction by Context, Extended Conventional DNN, Bidirectional Communication Between Layers, Mirror Neuron and Category Theory

\section{Introduction}

A function that even animals in the early stages of evolution have is feeding behavior that seeks food while avoiding danger. If the function is similar to that of zooplankton, it can be realized by electronic work of junior high school students, and the number of logic elements used may not be so different from the number of nerve cells in an actual organism. Whether or not the function is considered intelligent, it is an essential time-series data processing function even in animals that have evolved since then. In other words, it can be said that animals have evolved due to the increase in scale and the complexity of connections based on the nervous system that performs simple time-series data processing. In addition, it is known from neuroscience that the brain is composed of a combination of elemental circuits keeping the same structure while evolutionary process [1].

In this paper, a neural network with a learning function based on Hebb's law is presented as an elemental circuit of the brain, and is called a Basic Unit. The Basic Unit is made up of circuits that process time-series data and operates as a part of the whole neural network. The Basic Units are arranged in layers, and the information between the layers is bidirectional. The biggest feature of arranging in layers is that while processing of time series data, the activated part changes according to the context structure specific to the data, predicts mathematically the event from the context of the learned behavior, and do best of it. Learning function called conditioned reflexes and operant learning in behavioral psychology can be described by the behavior of neural networks that combine basic units.

Furthermore, in Chapter 3 also mentions imitation and the ability to symbolize objects. Imitation behavior is a behavior in which the movements of friends are reflected in their own recognition and operation. It is the basis of social life and ethics in the flock. In the imitation function, the function of a 
set of neurons called mirror neurons is drawing attention. The behavior of mirror neurons is based on existence of sets performing isomorphic behavior in the neural network lead to the symbolization of the object. Symbols have evolved to language from scream, warning, motion and etc. The ability to symbolize is an indispensable ability to realize advanced intelligence such as information transmission.

Two neural networks are introduced to solve the problem of how symbols should be treated in neural networks. One is a neural network that shows the behavior of symbols, and the other is a neural network that shows the behavior of images corresponding to symbols, both of which are related by long-term memory. These neural networks are placed above the neural networks derived from the basic behaviors of animals shown in Chapter 2, and scheme of operation of neural networks is shown.

\section{Basic Unit: As Universal Parts of Neural Network}

Eating behavior is an essential function in animals that have evolved to some extent and consists of a time series of multiple behaviors. That is, the process is to extend the arm toward the object, open the palm when approaching the object, close the palm when touching the object, grab the object and bring it closer to one's mouth. This operation can be realized by a circuit that is a slightly developed version of "electronic work at the junior high school level" [2]. As an example, let a circuit that moves the arm as shown in Figure 1.

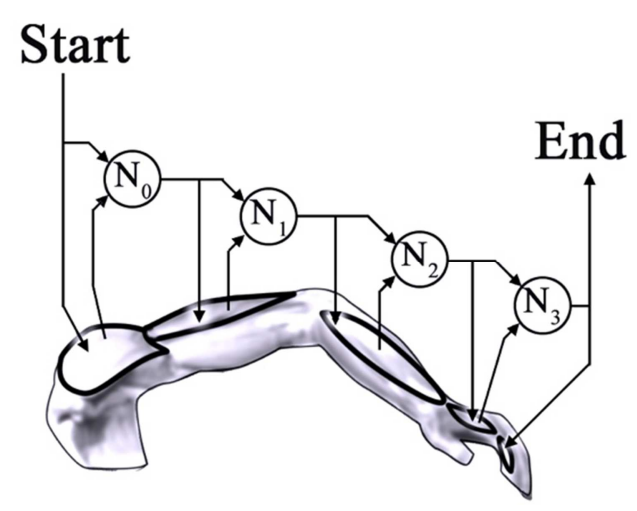

Figure 1. Muscles and nerves when grabbing things.

Here, there are circuits in which neurons having a function equivalent to the AND gate of an electronic circuit is connected in series, and each neuron is connected to the muscle of each part of the arm. When neuron $\mathrm{N}_{0}$ receives the start signal, the muscles of the shoulder get conditions such as muscle relaxation, load magnitude, joint bending angle, etc. (for simplicity, it is assumed various conditions are encoded and transmit). If the condition is matched toward to next step, signal to neuron $\mathrm{N}_{1}$ corresponding to upper arm is send. It continues until the output of the neuron $\mathrm{N}_{3}$ corresponding to the finger is completed.

Muscles that move intentionally, such as the muscles of the arm, are called voluntary muscles and are moved by pairing contraction signals and the responses. Since the directionality can be continuously determined by the ratio of each direction such as axons, the generality is not lost even if all the neural networks mentioned below are defined as bidirectional. It can be considered that the input from the bottom performs serial-parallel conversion (with waiting for input), and the input from above performs parallel-serial conversion (with waiting for response).

On the other hand, it has a structure that can be regarded as an extension of the conventional neural network that has been developed mainly for pattern recognition. First, as shown in Figure 2, any time series data composed of a finite number of elements can be divided into a plurality of subsequences in which the same element does not occur multiple times. Since the divided subsequences can be regarded as elements of new time series data in the upper hierarchy, it can be said that any time series data contains a hierarchy context.

\section{$c_{1} c_{7} c_{4} c_{6} c_{6} c_{0} c_{6} c_{5} c_{1} c_{3} c_{7} c_{8} c_{9} c_{9} c_{9} c_{7} c_{5} c_{4} c_{1}$ $\left|c_{1} c_{7} c_{4} c_{6}\right| c_{6} c_{0}\left|c_{6} c_{5} c_{1} c_{3} c_{7} c_{8} c_{9}\right| c_{9}\left|c_{9} c_{7} c_{5} c_{4} c_{1}\right|$}

Figure 2. Any time series data can be divided into substrings.

Since the divided subsequences can be regarded as elements of new time series data in the upper hierarchy, it can be said that any time series data contains a hierarchy context.

Consider a set $\mathrm{U}$ of elements equivalent to an AND gate as shown on the left in Figure 3.

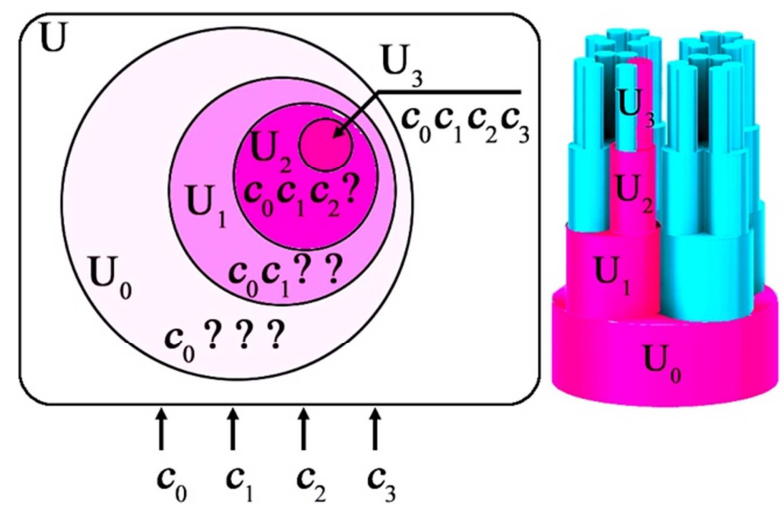

Figure 3. Time series data is recognized by elements arranged cylindrically.

Receives $\mathrm{c}_{0}, \mathrm{c}_{1}, \mathrm{c}_{2}, \mathrm{c}_{3}$, which are elements of time series data from the bottom. Each element is loosely coupled to the input, and the coupling state is not uniform. The operation of $\mathrm{c}_{0}, \mathrm{c}_{1}$, $\mathrm{c}_{2}$, and $\mathrm{c}_{3}$ will be explained below as received data, but the flow of operation to output time series data $c_{0}, c_{1}, c_{2}$, and $c_{3}$ is the same. $U_{0}$ is a set of elements activated by receiving the signal $\mathrm{c}_{0}$ that starts a series of operations. When $\mathrm{c}_{1}$ is received following $\mathrm{c}_{0}$, the element with high activity is a set of elements that are activated by the reception of $c_{0}$ one step before in addition to $c_{1}$. That is, the element that accept $c_{0} c_{1}$ is a set of $U_{1}$. The set of highly active elements is narrowed and narrowed until the last data $c_{3}$ is received, and the last remaining set of 
elements $\mathrm{U}_{3}$ becomes a pattern showing the time series data $\mathrm{c}_{0} \mathrm{c}_{1} \mathrm{c}_{2} \mathrm{c}_{3}$. The right side of Figure 3 is a diagram showing a neural network that processes time series data starting with $\mathrm{c}_{0}$ as a set of cylinders, and is called a Basic Unit. By setting the coupling coefficient between the Basic Units, the Basic Unit that is mostly input or mostly output and the Basic Unit that specializes only in the transmission of information (equivalent to a long axon in the nervous system) are also defined. This structure may be not so different to the structure of a hypothetical module called mini- and macro-columns, barrels, stripes, or blobs in neuro science [3].

All time-series data can be processed by arranging Basic Units for the number of elements displayed at the beginning of the time-series data and providing a hierarchy that regards their output as new time-series data. There are several possible realizations by neural networks, but one example is shown. After the direction of conversion is determined by which layer the input level is higher, the same state transition is performed. Input / output in one Basic Unit $U$ in layer $j$ is defined as follows.

$$
\begin{gathered}
H j s=\sum_{n=1}^{s} w_{k n} S_{k n} \\
H j p=\sum_{m=1}^{p} w_{i m} P_{i m}
\end{gathered}
$$

Here, $H_{j s}$ is the input via the coupling coefficient $w_{k n}$ from the Basic Unit $\mathrm{U}_{k n}$ in the layer $k$ one above $j$, and $S_{k n}$ is activity of serial output of $\mathrm{U}_{k n}$. And $H_{j p}$ is the input via the coupling coefficient $w_{i m}$ from the Basic Unit $\mathrm{U}_{i m}$ in the layer $i$ one below $j$ and $P_{i m}$ is activity of parallel output of $\mathrm{U}_{i m}$. The coupling coefficient $w$ changes depending on the activity of the Basic Unit connected to both ends. The higher the activity of the both Units, the larger the coefficient. This is a learning function included in Hebb's law.

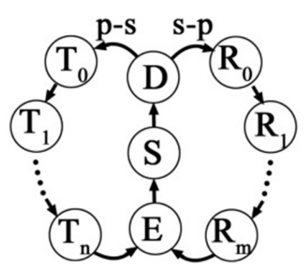

Figure 4. Basic unit state transition diagram.

The following is a description of each state in Figure 4. State $\mathrm{S}$ is standby state. Among the sum of the inputs from the basic units in the upper and lower layers represented by the equations (1) and (2) is both below the threshold value T, sate remains S. If either (1) or (2) exceeds $\mathrm{T}$, the state shifts to D and the conversion direction is determined. If $H_{j p}>\mathrm{T}>H_{j s}$, parallel serial conversion is activated and serial data is output to the lower layer in the states $T_{0}, T_{1}, ., T_{n}$. On the other hand, if $H j s>\mathrm{T}>H j p$, the serial data is received from the lower layer in the states $\mathrm{R}_{0}, \mathrm{R}_{1}, . ., \mathrm{R}_{\mathrm{m}}$, and the conversion result is output to the upper layer. In both conversions, when the conversion of the last data is completed, the end signal is transmitted toward the upper layer [4]. This state is E and next returns to the initial standby state $\mathrm{S}$. Even time series data with a deep context can be processed by arranging Basic Units in layers. The structure of neural network capable of such processing may be shown in Figure 5 [5-7].

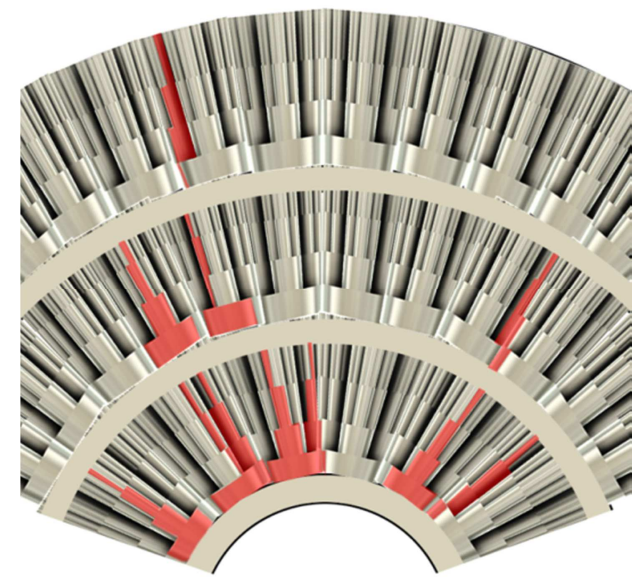

Figure 5. Basic Units are arranged in layers.

Figure 6 shows the correspondence between the changes in the activation region of the basic units arranged hierarchically and the context structure of the time series data [8].

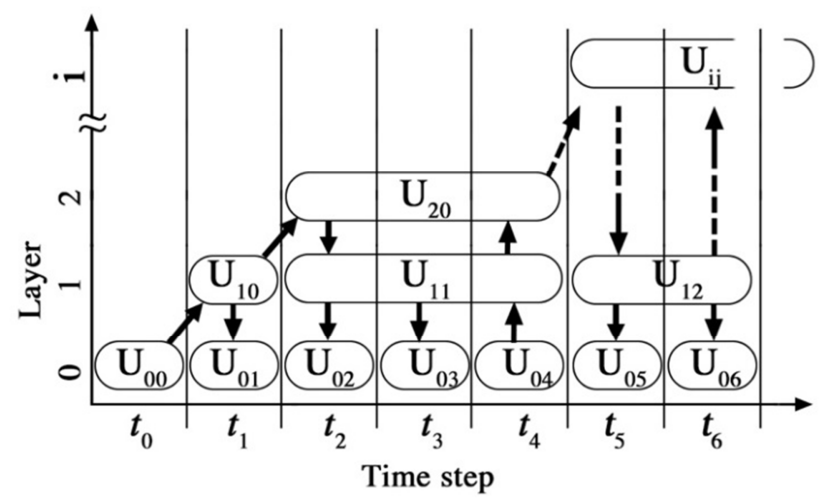

Figure 6. According the context of time series data Basic Units on active are changing while processing.

The bottom layer corresponds to units involved in movement such as sensory organs and muscles. It can be said that it is an extension of the neural network that has been developed for the purpose of image recognition. In the Figure 6 , the processing performed at times $t_{5}$ and $t_{6}$ is not an extension of the processing from $t_{0}$ to $t_{4}$. Processing $t_{5}$ to $t_{6}$ is activated and executed by the set of Basic Units $\mathrm{U}_{i j}$ at a higher level (indicated by the dashed arrow). In other words, the process from $t_{0}$ to $t_{6}$ is a story that includes the process from $t_{0}$ to $t_{4}$ as a prologue in the context. It may be useful to explain operant learning in behavioral psychology. Animals with developed brains store complex stories in multi-layered nervous tissue and live while making use of them to solve living problems [9].

It is assumed that the types of elements that make up time series data are finite. The intention of the assumption is that the resolution of the sensory organs of animals including us is limited, and the judgement or movement are accompanied by inaccuracy. Therefore, even if they make mistakes or the work is inaccurate, they have overcome it by using tools. In addition, 
although the length of time series data is also limited, it does not become an essential problem due to the hierarchy processing. Considering the process of recognizing symbols including the number of syllables and kanji in spoken language, the data length would not need to over 10. In other words, these assumptions are natural for theoretically looking at the intellectual behavior of animals. Voluntary muscles are accompanied by bidirectional information processing in which the results of the operation are paired, as in the example given above. However, the neurons involved in manipulation and outcome are not always adjacent. The part of the brain that controls vocal and the part of the brain that recognizes voice is separated, and when the connection between the two is impaired due to brain damage, aphasia and agnosia occur. These language disorders occur only in humans and may be related to the evolutionary sequence of animals.

\section{To Get Further Intelligence}

\subsection{Imitation and Analogy}

In the previous chapter, it was shown that a neural network that processes time-series data can be constructed by connecting parts (Basic Units) that basically have the same structure in layers. The neural network predicts an event by using the fact that the activated region in the neural network changes with processing according to the context structure inherent in the time series data, and can take the optimum measure after trial and error. The logic was developed based on the Hebb law, but even if a system is assembled by combining semiconductors, complicated operations can be realized simply by arranging modules with basically the same structure, so it is industrially useful in terms of both hardware and software. It may be possible to build a system.

The goal of this chapter is not to develop a robot that is required to efficiently perform a given task, but to elucidate the essence of intelligence along with the evolution of animals. There are problems on the neural network which has been developed around the pattern recognition function, are not familiar with the performances that animals such as familiar pets naturally do. One of them is the imitation function. Many insects and fish also have a mimicking function. The cry raises the identity of the group, and even small fish threaten predators by gathering and swimming in a form reminiscent of giant creatures. Experiments with monkeys have shown that a region of the brain called mirror neurons plays a demanding role. It is said that imitation not only enables collective action, but also makes it possible to replace the situation of peers with oneself and to infer it, which is said to be the seed of ethics. Marco Iakaboni explained as follows [10].

If a cell discharged more vigorously while the monkey was grasping the food in order to eat, that same cell discharged more vigorously while the monkey was observing the human experimenter grasping the food in order to eat.

The explanation of Iakaboni can be expressed using the neural network shown as follows.

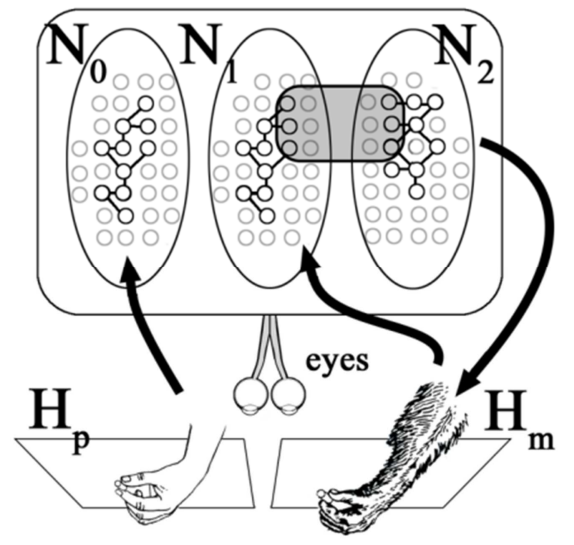

Figure 7. Conceptual diagram of mirror neuron behavior.

In the Figure 7, when the monkey extends his hand $\mathrm{H}_{\mathrm{m}}$ to food, the neural network $\mathrm{N}_{1}$ that observes $\mathrm{H}_{\mathrm{m}}$ and the neural network $\mathrm{N}_{2}$ that controls the movement of $\mathrm{H}_{\mathrm{m}}$ are activated. This is because the muscles that move the hand are voluntary muscles, so the movement and the feeling of the result are always paired to perform a series of processes. On the other hand, when the experimenter moves the hand $\mathrm{H}_{\mathrm{p}}$ to food in front of the monkey's eyes, $\mathrm{N}_{1}$ is activated along with the neural network $\mathrm{N}_{0}$ that is observing the $\mathrm{H}_{\mathrm{p}}$. As mentioned above, $\mathrm{N}_{1}$ is activated by the movement of the monkey's own hand and the behavior of $\mathrm{N}_{1}$ is very similar to the behavior of $\mathrm{N}_{0}$. As a result, $\mathrm{N}_{1}$ is activated via $\mathrm{N}_{0}$, and even the neural network $\mathrm{N}_{2}$ that moves the monkey's own hand is activated. In this way, the situation of the companion in front of you is naturally transmitted. Various stories will unfold depending on the relationship between you and the other party.

What is important in the above explanation is the isomorphism of the behavior between neural networks. The idea of category theory is useful for formulating the behavior of a set of Basic Units [11-13].

\subsection{Long-term Memory and Mutual Recognition Sharing}

In this paper, imitation behavior is regarded as the evolution of animal intellectual ability following feeding behavior, and it is reflected in the neural network. But intelligence must evolve towards further goals. What is the essence of intelligence that characterizes us humanity? Let's compare it with other animals. As a familiar example, let's take a pet with some tricks. It is only in relation to the environment in which the pet is placed that the pet understands the owner's instructions. Even if you scold or praise what your pet did the day before, your pet will not understand it, and you cannot promise to do it the next day. Perhaps many of the animals around us are aware of daily sunshine changing and even the changing climate each year, and may be aware of time-series data of lengths that fit their life cycle, such as food and hibernation preparation. But they can't tell the story to their peers. They don't have the means to express nor understand the story.

On the other hand, we ponder while watching the sun setting on the horizon and the rising stars, what happened on 
that day, expectations for tomorrow, and even the future of the Earth and the universe, and to our friends those thoughts are told or expressed as a work of art. In other words, we can create a story of any length starting from the "space-time pointer" that can be set arbitrarily, and share the story with our friends [14].

The following logic development is rather top-down process than explained so far. The neural network already shown targets the "real world" that can be operated by voluntary muscles based on the information from the sensory organs. The proposing new neural network targets "descriptive world" and is connected on the conventional neural network that targets "real world" [15].

The $N_{R}$ drawn at the bottom of the Figure 8 is the neural network already shown, and is a model of the basic part of the animal's brain that lives by manipulating events in the area that can be recognized by the sensory organs using limbs etc. Upper $\mathrm{N}_{\mathrm{I}}$ and $\mathrm{N}_{\mathrm{L}}$ are neural networks added to realize the functions that more evolved animal brains would have. The interaction of each neural network is explained below, and it is shown that the proposed neural network can be a mathematical object based on neuroscience. $\mathrm{N}_{\mathrm{L}}$ has the symbolic events handled by $\mathrm{N}_{\mathrm{R}}$, that is, shouts, gestures,.., the names of things, and the elements (including the so-called "5W1H") that make up the context that connects them. The image in the $\mathrm{N}_{\mathrm{R}}$ captured by the sensory organs is stored in $\mathrm{N}_{\mathrm{I}}$. It can be understood that $\mathrm{N}_{\mathrm{L}}$ is a neural network that handles languages and $\mathrm{N}_{\mathrm{I}}$ is a neural network that handles images. $\mathrm{N}_{\mathrm{I}}$ and $\mathrm{N}_{\mathrm{L}}$ do not always work in sync with $\mathrm{N}_{\mathrm{R}}$, but they receive information from the $\mathrm{N}_{R}$ via the LMS, and the content is added / corrected to maintain consistency with the $\mathrm{N}_{\mathrm{R}}$. LMS is abbreviation of Long-Term Memory Synthesizer (tentative name) and is the virtual nervous system that probably contains the hippocampus.

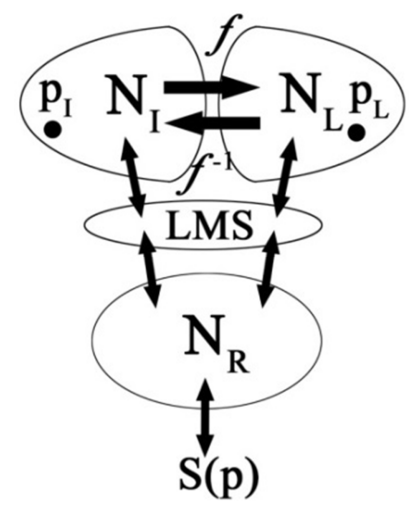

Figure 8. A processing scheme of the brain oriented neural networks with long-term memory and language ability.

These neural networks are collectively expressed as $B=\left(N_{R}\right.$, $\mathrm{N}_{\mathrm{L}}, \mathrm{N}_{\mathrm{I}}$ ). The basic operation of the above neural networks is the same as $N_{R}$, which is not far from the knowledge of neuroscience. In short, these neural networks can be neuroscience-based mathematical objects, unlike the items and software modules presented top-down simply for the depiction of psychological activity.
(1) $\mathrm{N}_{\mathrm{L}}$ and $\mathrm{N}_{\mathrm{I}}$ can be isomorphic. There is a set of active basic units in $\mathrm{N}_{\mathrm{I}}$, and the basic unit at the start of the state transition is named the space-time pointer $\mathrm{p}_{\mathrm{I}}$. The movement of the activated part of $\mathrm{N}_{\mathrm{I}}$ starting with $\mathrm{p}_{\mathrm{I}}$ is reflected in $\mathrm{N}_{\mathrm{L}}$. The space-time pointer $\mathrm{p}_{\mathrm{L}}$ corresponding to $\mathrm{p}_{\mathrm{I}}$ is also in $\mathrm{N}_{\mathrm{L}}$, and the movement of the activated part starting with $\mathrm{p}_{\mathrm{L}}$ is called story $S(p)$. $S(p)$ is represented by letters, symbols, gestures and voice. The story $\mathrm{S}(\mathrm{p})$ left in the past can be read into $\mathrm{N}_{\mathrm{L}}$ via $\mathrm{N}_{R}$ and reflected in $\mathrm{N}_{\mathrm{I}}$. If the state transition brought to $\mathrm{N}_{\mathrm{I}}$ as a result of reading is the same as the state transition of $\mathrm{N}_{\mathrm{I}}$ when $\mathrm{S}$ (p) is generated, then between the mapping $f$ from $\mathrm{N}_{\mathrm{L}}$ to $\mathrm{N}_{\mathrm{I}}$ and its inverse mapping $f^{1}$. It can be expressed that $f \circ f^{1}=1$ hold, and it is expressed as $\mathrm{N}_{\mathrm{L}} R \mathrm{~N}_{\mathrm{I}}$. If it is $\mathrm{N}_{\mathrm{L}} R \mathrm{~N}_{\mathrm{I}}$, it is obviously $\mathrm{N}_{\mathrm{I}} R \mathrm{~N}_{\mathrm{L}}[16]$.

(2) Assume sets of neural networks $B=\left(N_{R}, N_{L}, N_{I}\right)$ and $\mathrm{B}^{\prime}=\left(\mathrm{N}_{\mathrm{R}}^{\prime}, \mathrm{N}_{\mathrm{L}}^{\prime}, \mathrm{N}_{\mathrm{I}}^{\prime}\right)$. When there are stories $\mathrm{S}(\mathrm{p})$ and $\mathrm{S}\left(\mathrm{p}^{\prime}\right)$, and $\mathrm{S}(\mathrm{p})=\mathrm{S}\left(\mathrm{p}^{\prime}\right)$ and $\mathrm{N}_{\mathrm{L}}^{\prime} R \mathrm{~N}_{\mathrm{I}}^{\prime}$ holds, then $\mathrm{N}_{\mathrm{I}} R \mathrm{~N}_{\mathrm{I}}^{\prime}$. It can be said that $\mathrm{B}$ and $\mathrm{B}^{\prime}$ can have a common image via $\mathrm{S}(\mathrm{p})$, and this state is expressed as $\mathrm{B} C \mathrm{~B}^{\prime}$. If it is $\mathrm{B} C \mathrm{~B}^{\prime}$, it is clear that $\mathrm{B}$ ' $C \mathrm{~B}$ holds.

(3) Let $\mathrm{S}_{\mathrm{i}}$ be the set of neural network sets $\mathrm{B}_{\mathrm{n}}$ for which $\mathrm{B}_{\mathrm{i}} C$ $\mathrm{B}_{\mathrm{n}}$ holds for the neural network set $\mathrm{B}_{\mathrm{i}}$. $\mathrm{B}_{\mathrm{j}} C \mathrm{~B}_{\mathrm{k}}$ holds for any $\mathrm{B}_{\mathrm{j}}$, $B_{k}$ belonging to $S_{i}$.

The above definition may not represent the realization of quantification of physical states within a neural network, but only the states that may be retained at key points in the behavior of the entire neural network. In the first place, it is impossible to measure the presence or absence of cognition $(R)$, mutual understanding $(C)$, and sociality $(S)$ while observing the active brain. However, it is useful at least for a short period of time to recognize from the behavior of the other person that there is the above relationship $R, C, S$ with the other person. Unfortunately, in our society, personal misunderstandings and false behaviors hide the true situation, and from a certain point the whole situation can change dramatically.

\section{Conclusion}

It has been said in neuroscience that the brain must be composed of a combination of neural modules with similar functions. In this paper, a module called Basic Unit is proposed as a basic component of a neural network, and it is shown that general time-series data can be processed hierarchically. A neural network using a Basic Unit is an extension of the structure of a neural network developed from conventional pattern recognition means. The functionality of the module will need to be examined from both the neuroscience side and the electronics side (quick way is to use microcomputer). In the process of examining the Hebb's law which is the basis of this paper new idea of electronic circuits may be expected [17].

In Chapter 3, in order to realize advanced intelligence, two neural networks that deal "descriptive world" are set up above the neural network that deals "real world". Although the scheme of operation between neural networks is shown, there are many problems in realizing the function, and not only 
neuroscience and electronics but also psychology, linguistics and philosophical viewpoints are required. In particular, the theme of the language is vast and profound.

If multiple intelligent individuals gather, they not only ask questions and respond to each other, but also transmit, absorb, correct, and deny knowledge through dialogue using "5W1H" and create a culture in the group. Isn't it one of the goals of artificial intelligence to build a group with a common understanding?

\section{References}

[1] D. C. Dennet, "From Bacteria to Bach and Back: The Evolution of Minds", In Penguin Books, 2018.

[2] Paolo Arena, Marco Cali, Luca Patane, Agnese Portera, Roland Strauss SC, "Modelling the insect Mushroom Bodies: Application to Sequence learning", Neural Networks 67 (2015) 37-53.

[3] Gyorgy Buzsaki, "Rhythms of the Brain", Oxford University Press, 2006, p43 Networks and Applications, Volume 6, Issue 1, June 2020.

[4] Qianli Ma, Wanqing Zhuang, Lifeng Shen, Garrison W. Cottrell, "Time series classification with Echo Memory Networks", Neural Networks 117 (2019) 225-239.

[5] L. Andrew Cowaed, Ron Sun, "Hierarchical approaches to understanding consciousness", Neural Networks 117 (2019).

[6] Hoon Keng Poon, Wun-She Yap, Yee-Kai Tee, Wai-Kong Lee, Bok-Min Goi "Hierarchical gated recurrent neural network with adversarial and virtual adversarial training on text classification”, Neural Networks 119 (2019) 299-312.
[7] Ann Sizemore, Chad Giusti, Ari Kahn, Richard F. Betzel, Danielle S. Bassett "Cliques and Cavities in the Human Connectome", Cornell University arxiv.org/abs/1608.03520, 2016.

[8] Hiroshi Yamakawa, "Attentional Reinforcement Learning in the Brain", New Generation Computing, January 2020.

[9] S. Yanagawa. 2017, "Learning Machine That Uses Context Structure to Search Policy", https://jsai.ixsq.nii.ac.jp/. SIG-AGI-007-07.

[10] T M. Iacoboni, "Mirroring People", Picador 33. 2008, Chapter 4, p106.

[11] S. Yanagawa "Each Role of Short-term and Long-term Memory in Neural Networks", American Journal of Neural Networks and Applications, Volume 6, Issue 1, June 2020.

[12] T. Leinster "Basic Category Theory", Cambridge University Press 2017.

[13] S. Yanagawa "A neural network that processes time series data in which element data is linked to symbols", Third International Workshop on Symbolic-Neural Learning (SNL-2019) Poster presentation.

[14] S. Dehaene, Consciousness and Brain Penguin Books, 2014.

[15] Michael j. Healy, Thomas P. Caudill, "Episodic memory: A hierarchy of spatiotemporal concepts", Neural Networks 120 (2019) 40-57.

[16] W. J. Freeman "How Brains Make up Their Minds" Weidenfeld \& Nicolson Ltd. 1999.

[17] Kenji Doyaa \& Tadahiro Taniguchi, "Toward Evolutionary and Developmental Intelligence", Current Opinion in Behavioral Sciences Volume 29, October 2019, Pages 91-96. 